\title{
Reduced field-of-view stack-of-spirals enables high spatiotemporal resolution 3D perfusion imaging
}

Yang Yang ${ }^{1 *}$, Li Zhao ${ }^{5}$, Xiao Chen ${ }^{4}$, Kelvin Chow ${ }^{2}$, Peter W Shaw ${ }^{2}$, Jorge A Gonzalez ${ }^{2}$, Frederick H Epstein ${ }^{1,3}$, Craig H Meyer ${ }^{1,3}$, Christopher M Kramer ${ }^{2,3}$, Michael Salerno ${ }^{2,3}$

From 19th Annual SCMR Scientific Sessions

Los Angeles, CA, USA. 27-30 January 2016

\section{Background}

First-pass contrast-enhanced myocardial perfusion CMR is a useful non-invasive technique for evaluating coronary artery disease. 3D perfusion imaging provides images at the same cardiac phase which may be advantageous for quantifying ischemic burden, but current techniques have limited spatiotemporal resolution. We previously presented a 3D stack-of-spiral (SoS) perfusion method, however this technique had a long temporal footprint $(240 \mathrm{~ms})$ and thick slices $(8 \mathrm{~mm})$. As the heart occupies

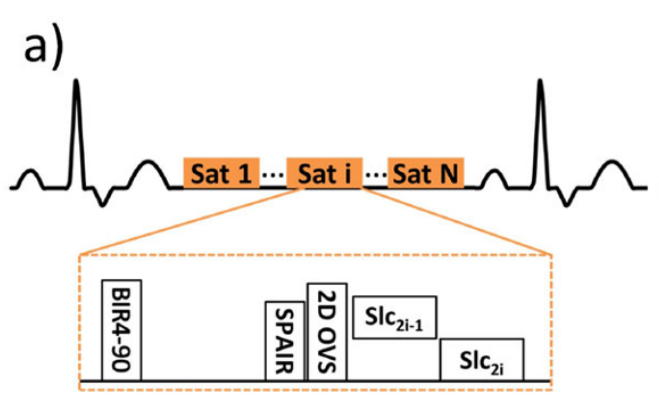

b)

c)

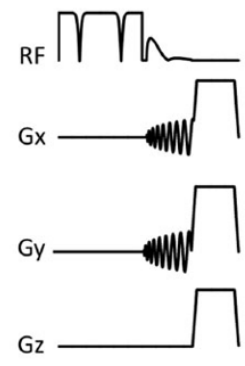

d)

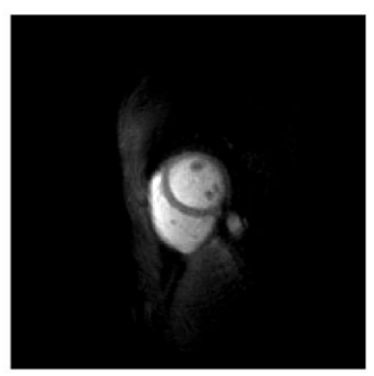

e)

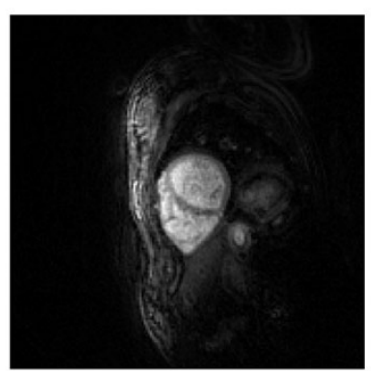

Figure 1 a) 2D single-shot spiral perfusion sequence with outer volume suppression (OVS). b) 3D stack-of-spirals (SoS) perfusion sequence with OVS and centric ordering. c) OVS preparation consisted of a BIR4 tip-down, a 2D spiral tip-back and a spoiler. 2D (d) and 3D (e) OVS perfusion images show attenuation of signal outside of the heart region.

'Biomedical Engineering, University of Virginia, Charlottesville, VA, USA

Full list of author information is available at the end of the article 

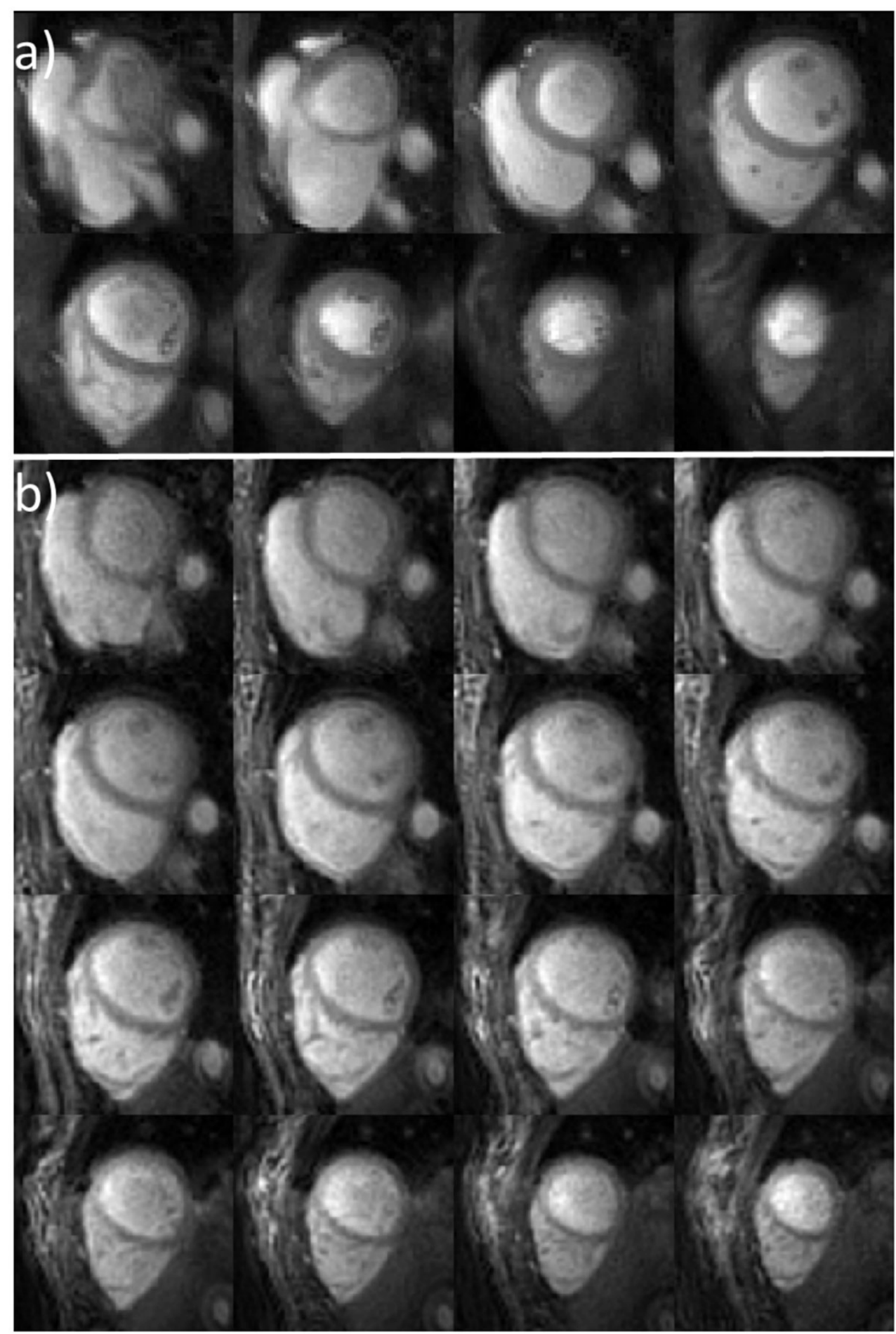

Figure 2 Rest perfusion images with whole heart covearge at middle time frame from the same subject using the 2D single-shot spiral perfusion sequence with OVS (a) and 3D stack-of-spirals sequence with OVS (b).

only a small portion of the chest, reduced FOV (rFOV) techniques enable imaging over a small FOV with substantially improved sampling efficiency. We recently demonstrated a 2D outer volume suppressed (OVS) single-shot spiral perfusion sequence with a temporal resolution of $8 \mathrm{~ms}$ per image and whole heart coverage with $2 \mathrm{~mm}$ resolution. We hypothesized that application of OVS to 3D SoS perfusion imaging could reduce the 
temporal footprint while increasing the through-plane spatial resolution.

\section{Methods}

An OVS preparation was incorporated into a 2D singleshot (Figure 1a) and 3D SoS (Figure 1b) perfusion sequences. The OVS consisted of a non-selective adiabatic BIR-4 tip-down pulse, a 2D spiral spatially selective tip-back pulse and a spoiler to suppress signal outside the heart (Figure 1c). 2D and 3D first-pass perfusion were performed with a $0.075 \mathrm{mmol} / \mathrm{kg}$ Gd-DTPA bolus, separated by 20 min contrast washout time, in 6 subjects on a $1.5 \mathrm{~T}$ Avanto Siemens scanner. 2D sequence parameters included: FOV $170 \mathrm{~mm}$, TE $1.0 \mathrm{~ms}$, TR $9 \mathrm{~ms}$, SRT $80 \mathrm{~ms}$, FA $90^{\circ}, 8 \mathrm{~ms}$ per slice, 8 slices with $8 \mathrm{~mm}$ thickness, $2 \mathrm{~mm}^{2}$ in-plane resolution. 3D sequence parameters were similar except: SRT $150 \mathrm{~ms}$, FA $35^{\circ}$, acquisition time $180 \mathrm{~ms}, 20$ slices with $4 \mathrm{~mm}$ thickness. The images were reconstructed using Block LOw-rank Sparsity with Motion guidance (BLOSM) combined with SENSE.

\section{Results}

Better outer volume suppression was observed for the $2 \mathrm{D}$ technique as compared to the $3 \mathrm{D}$ technique (Figure 1d-e) due to its shorter acquisition time. However, in both cases, OVS significantly attenuated signal outside of the heart, reducing spatial aliasing artifacts for both $2 \mathrm{D}$ and $3 \mathrm{D}$ techniques. Figure 2 shows 2D single-shot and 3D SoS perfusion images from the same subject at a similar time point. Both the 2D and 3D techniques resulted in good image quality. Given the very short temporal footprint of the 2D single-shot technique, fine details of the cardiac trabeculae and papillary muscles are better resolved than with the 3D technique. 3D SoS provided high through-plane resolution $(4 \mathrm{~mm})$ reducing partial volume effects and provided better depiction of the apical slices, but with some loss of fine detail due to the longer temporal footprint. Similar image quality was found for the other cases.

\section{Conclusions}

We demonstrated the successful application of OVS to 3D SoS perfusion techniques. The improvement of sampling efficiency using OVS enables 3D imaging with a combination of high in-plane and through-plane spatial resolution and a temporal footprint of $180 \mathrm{~ms}$.

\section{Authors' details}

'Biomedical Engineering, University of Virginia, Charlottesville, VA, USA. ${ }^{2}$ Medicine, University of Virginia, Charlottesville, VA, USA. ${ }^{3}$ Radiology, University of Virginia, Charlottesville, VA, USA. ${ }^{4}$ Siemens Medical Solutions, Princeton, NJ, USA. ${ }^{5}$ Beth Israel Deaconess Medical Center, Harvard Medical School, Boston, MA, USA.
Published: 27 January 2016

doi:10.1186/1532-429X-18-S1-P325

Cite this article as: Yang et al:: Reduced field-of-view stack-of-spirals enables high spatiotemporal resolution 3D perfusion imaging. Journal of Cardiovascular Magnetic Resonance 2016 18(Suppl 1):P325.
Submit your next manuscript to BioMed Central and take full advantage of:

- Convenient online submission

- Thorough peer review

- No space constraints or color figure charges

- Immediate publication on acceptance

- Inclusion in PubMed, CAS, Scopus and Google Scholar

- Research which is freely available for redistribution

Submit your manuscript at www.biomedcentral.com/submit 